University of Wollongong

Research Online

Faculty of Engineering - Papers (Archive)

Faculty of Engineering and Information

Sciences

2009

\title{
Multi-jointed robot finger driven by artificial muscle actuator
}

Nguyen Huu Chuc

Sungkyunkwan University

Jong Kil Park

Sungkyunkwan University

Nguyen Huu Lam Vuong

Sungkyunkwan University

DukSang Kim

Sungkyunkwan University

Ja Choon Koo

Sungkyunkwan University

See next page for additional authors

Follow this and additional works at: https://ro.uow.edu.au/engpapers

Part of the Engineering Commons

https://ro.uow.edu.au/engpapers/5491

\section{Recommended Citation}

Chuc, Nguyen Huu; Park, Jong Kil; Vuong, Nguyen Huu Lam; Kim, DukSang; Koo, Ja Choon; Lee, Youngkwan; Nam, Jae-do; and Choi, Hyouk Ryeol: Multi-jointed robot finger driven by artificial muscle actuator 2009.

https://ro.uow.edu.au/engpapers/5491

Research Online is the open access institutional repository for the University of Wollongong. For further information contact the UOW Library: research-pubs@uow.edu.au 


\section{Authors}

Nguyen Huu Chuc, Jong Kil Park, Nguyen Huu Lam Vuong, DukSang Kim, Ja Choon Koo, Youngkwan Lee, Jae-do Nam, and Hyouk Ryeol Choi 


\title{
Multi-jointed robot finger driven by artificial muscle actuator
}

\author{
Nguyen Huu Chuc, Jong Kil Park, Nguyen Huu Lam Vuong, DukSang Kim, Ja Choon Koo, \\ Youngkwan Lee, Jae-do Nam, Hyouk Ryeol Choi
}

\begin{abstract}
In this paper we present a robotic actuation system by artificial muscle actuator based on dielectric elastomer. A novel linear actuator called "multi-stacked actuator" is presented, which can be embedded in the phalanges of the finger and ensures a compact design of the overall system. As an exemplary work, a two degree-of-freedom robot finger is developed and its performance is experimentally demonstrated. The proposed system can be extended to the multi-fingered robot hand easily, and applied even for articulated mechanisms such as legged robots etc.
\end{abstract}

\section{INTRODUCTION}

A wide variety of robot hands have been developed up to now, which are mostly driven by servomotors[1], [2], [3]. Although motors achieve acceptable performance, their major limitation is that they are voluminous, heavy and require complex transmission mechanisms. The above shortcomings are major sources of restricting robot hands solely in laboratory use. In fact, existing robot hands are relatively heavy compared to the human hand owing to the weight of motors and complex transmission mechanisms. As one of the most demanding applications, a recent survey on the satisfaction with prosthetic devices have reported that approximately $23 \%$ of the participants were dissatisfied with the weight of their prosthetic limb [4].

This research is aimed at overcoming the shortcomings of conventional actuators by utilizing an artificial muscle actuator. The emergence of active materials such as ElectroActive Polymers (EAPs) or Shape Memory Alloys(SMA) whose characteristics are similar to those of human muscles, have encouraged the development of the artificial muscle actuator and extended to robot hands actuated with it. Recently, several robot hands using the artificial muscle actuators have been developed[5], [6], [8], [7]. These actuators have advantages of lightweight without adding mechanical complexity. Also, EAP actuators have many attractive properties, typically soft, inexpensive, and fracture tolerant[9], [10]. Among them, dielectric elastomer actuators are one of the most prospective because of their large deformation and high force density, and cost effectiveness[11]. Actuators made from the dielectric elastomer have been applied in

This work was supported by the Intelligent Robotics Development, one of the 21 st Century Frontier R\&D Programs funded by the Ministry of Commerce, Industry and Energy.

N. H. Chuc, J. K. Park, N. H. L. Vuong, D. S. Kim, J. C. Koo, Y. K. Lee, J. D. Nam, H. R. Choi are with School of Mechanical Engineering, Sungkyunkwan University, Suwon, Kyunggi-do, Korea

Y. K. Lee, J. D. Nam are with School of Applied Chemistry and Chemical Engineering, Sungkyunkwan University, Suwon, Kyunggi-do, Korea

All correspondences are to be sent to Prof. Choi at hrchoi@me.skku.ac.kr the robots, micro devices, micro air vehicles, etc. So far, various configurations of actuators have been proposed such as planar, tube, roll extender, folding and stack, etc. [12], [13], [14], [15], [16], [18], [17], [19]. In the previous report, a new multi-stacked actuator using synthetic elastomer has been introduced by our group[19]. The proposed actuator has advantages for controlling each layer independently. In this research, we present a two DOF(degree-of-freedom) robot finger employing the multi stacked actuator. The multi stacked actuator is embedded in the link, hence, the finger becomes compact, simple in mechanism and lightweight. Its design and control issues are briefly addressed in this report.

The paper is organized as follows. In the first, a multi stacked actuator with trapezoidal actuator unit is introduced in section II. In section III, the design of the joint using the simple slider mechanism is described in details. In section IV describes the finger mechanism utilizing multi stacked actuator and the open-loop controller is mentioned in section V. Finally, the conclusions are included in section VI.

\section{MULTI-STACKED ACTUATOR}

\section{A. Actuation principle}

The operational principle of the dielectric elastomer elaborated in previous reports, is briefly introduced in this section [9], [10], [11]. When a voltage is supplied across a dielectric elastomer film coated with compliant electrodes on both sides, the film shrinks in thickness and expands in area accordingly as shown in Fig. 1. It is a kind of field-induced deformation and the electrostatic force by the charges on the surface of the film, called Maxwell stress, causes contraction of the film along the thickness direction. Based on simple electrostatic model, the effective pressure can be derived as follows.

$$
p=\varepsilon_{0} \varepsilon_{r}\left(\frac{V}{d}\right)^{2}
$$

where $p$ is electrostatic pressure (Maxwell stress), and $\varepsilon_{0}$, $\varepsilon_{r}$ mean the free-space permittivity $\left(8.85 \times 10^{-12} \mathrm{~F} / \mathrm{m}\right)$, and the relative dielectric constant of the elastomer, respectively. $V$ denotes the applied voltage and $d$ is the thickness of the film.

In the previous report, we have developed the multistacked actuator by using synthetic elastomer [19]. The proposed actuator is designed to be directly driven by Maxwell stress without using prestretching. In addition, it produces a relative large displacement(up to $10 \%$ of the effective length of the actuator) because the novel synthetic 


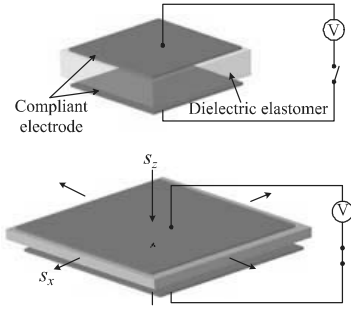

Fig. 1. Operational principle of dielectric elastomer actuator

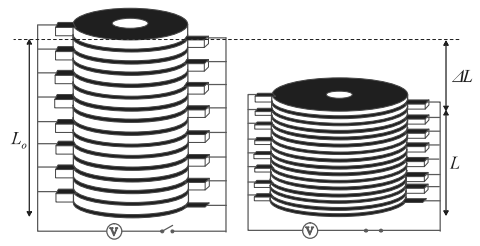

Fig. 2. The operation principle of multi-stacked actuator

elastomer is utilized. Its fundamental principle of operation is as shown in Fig. 2. When the voltage is applied between two electrode layers, Maxwell stress is produced, and thus the dielectric elastomer is compressed along the axial direction. The compression of each layer results the lateral expansion of the actuator because of the incompressibility of the polymer. Consequently, the deformation of the multi-stacked actuator is the sum of the deformation of each layer, and the total deformation is expressed as follows:

$$
x=\Delta L=\sum_{i=1}^{N} \Delta L_{i}=N \Delta L_{i}=N \times L_{a} \frac{\varepsilon_{0} \varepsilon_{r}\left(\frac{V}{d}\right)^{2}}{Y}
$$

where $Y, L_{a}$ are respectively the Young's modulus and the initial length of multi-stacked actuator.

The principle of multi-stacked actuator is simple but its performance is quite different depending on the design of the actuator. In particular, the geometry of the actuator unit has large influence on the performance of the actuator. In the previous work, two types of design: the circular actuator and the rectangular one have been designed.

\section{B. Trapezoid Multi-stacked actuator}

Recently, we proposed a folded actuator and compared actuation performances depending on the geometric shape of the actuator[19]. The folding, as shown in Fig. 8, makes a free edge, which enhances the deformation of the actuator because the boundary of non-active region obstructing the deformation of the active region is partially removed. According to simulation with ANSYS as shown in Figs. 3 and 4, the deformation of the active region reduces as the dimension of the inactive boundary becomes large. When the boundary length of the actuator increases, the compressed strain of a circular actuator decreases. Consequently, the non-active region should be minimized as small as possible and as the result, a trapezoidal one is proposed.
The deformation of circular, rectangular and trapezoid actuator are simulated by ANSYS, the deformation of trapezoid actuator is shown in Figs 5. The simulation is conducted when the inactive region dimension (boundary length) of actuator is $0.5 \mathrm{~mm}$, while the active region areas and thickness are similar to the circular actuator, respectively are $300 \mathrm{~mm}^{2}$, $200 \mu \mathrm{m}$. According to the simulation results, when three actuators are applied with the the same voltage (same the electric field $25 \mathrm{MV} / \mathrm{m}$ ), the trapezoid actuator has better performance than the rectangular and circular one as shown in Fig. 6. The experiment results are described in Fig. 7. Through the experiment results, it is noted that the folded trapezoid actuator has better performance than the others. Consequently, utilizing this actuator is very significant in robotic applications.

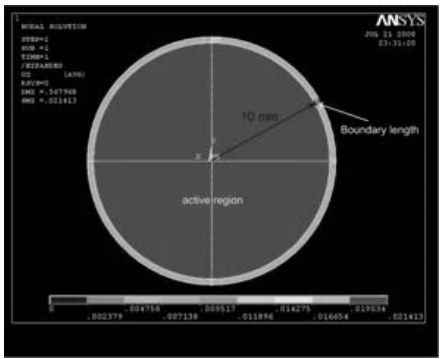

Fig. 3. Deformation of active region and inactive region

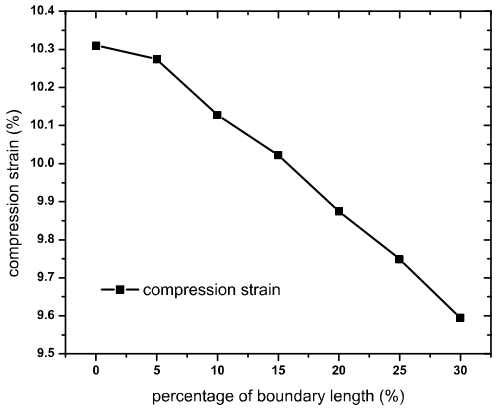

Fig. 4. The effect of the boundary on deformation of actuator layer.

The mechanism of the trapezoid actuator has been described in Fig. 8 and the prototype of this actuator is illustrated in Fig. 9. Firstly, the hexagonal pattern that is coated with electrode both side is created. Next, this pattern is folded to have one free edge. These folded actuators will be stacked to become multi-stacked trapezoid actuator. The developed multi-stacked trapezoid actuator has free stroke about $5 \%$ of inial length and it can lift a mass about $2 \mathrm{~kg}$.

\section{DESIGN OF THE JOINT}

\section{A. Joint mechanism}

The multi-stacked actuator can generate the linear motion like natural muscles without any mechanical substitute. Consequently, it is necessary to transfer the linear motion into the 


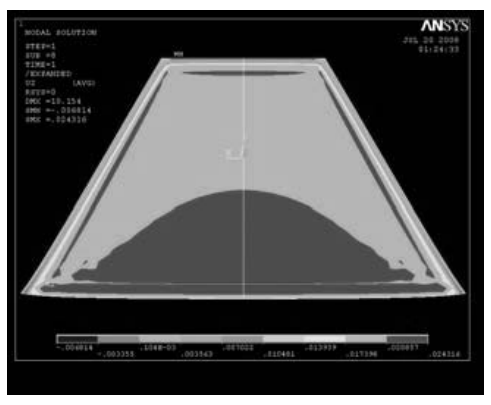

Fig. 5. The simulation of trapezoid actuator unit

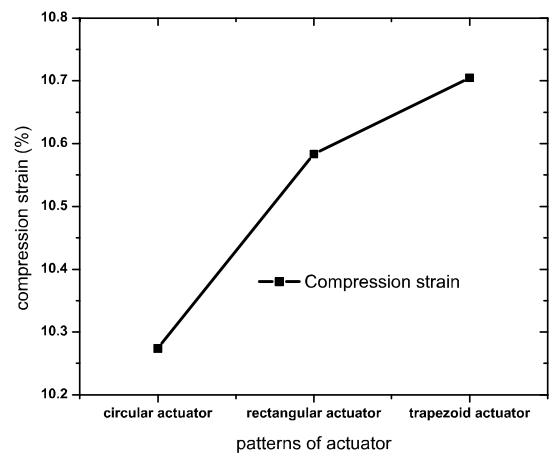

Fig. 6. Deformation of different patterns of actuator

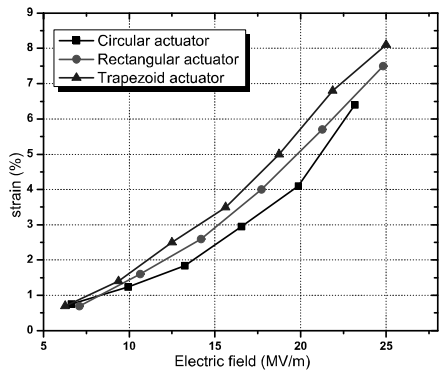

Fig. 7. Experimental results of circular actuator, rectangular actuator and trapezoid actuator.

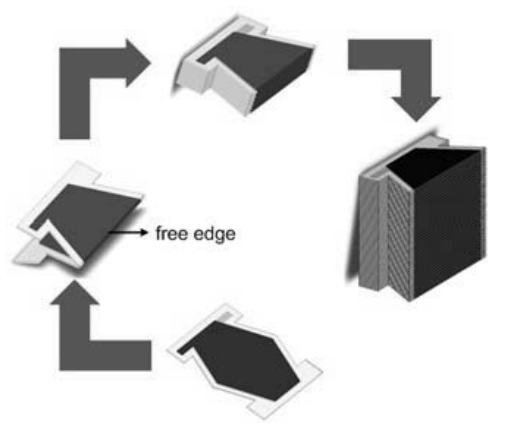

Fig. 8. The design of trapezoid actuator.

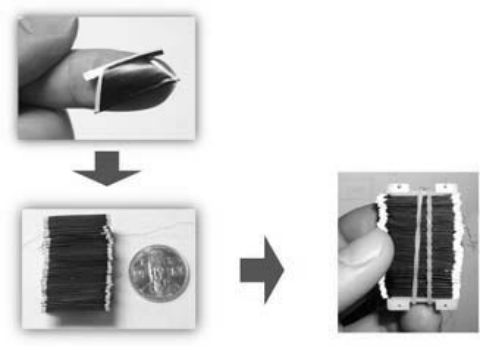

Fig. 9. The design of trapezoid actuator.

rotational one to apply in robot field. In this paper, a simple slider crank mechanism is chosen to convert the linear motion of multi-stacked actuator to rotational motion of crank. The active elastic force of the multi-stacked actuator causes the piston to translate along the vertical axis. This action causes the link rotate an angle $\theta$ as shown in Fig. 10.

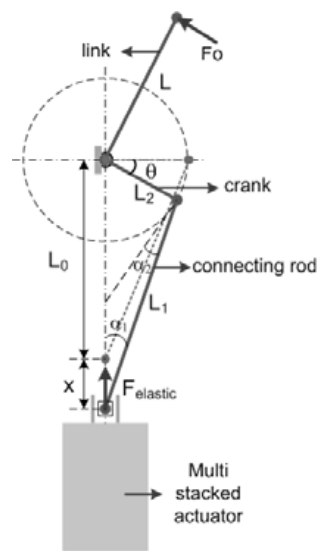

Fig. 10. Slider crank mechanism

\section{B. System analysis}

The purpose of this section is to determine the total output force and displacement of the link from the elastic force of the multi-stacked actuator.

1) Position analysis: When the driving voltage is applied, the multi-stacked actuator is compressed a length $x$, the link $L$ rotates an angle $\theta$ calculated as follows:

$$
\theta=\frac{\pi}{2}-\operatorname{arcos}\left(\frac{\left(L_{0}+x\right)^{2}+L_{2}^{2}-L_{1}^{2}}{2\left(L_{0}+x\right) L_{2}}\right)
$$

where $L_{1}$ and $L_{2}$ are the connecting rod and the crank radius, respectively. $L_{0}$ is the distance between the crank and the actuator when $\theta=0$.

2) Force analysis: The elastic force caused by the compression of the multi-stacked actuator is calculated by the Young's modulus $\left(E_{0}\right)$ of the synthetic elastomer material 
and the compression of the multi-stacked actuator:

$$
F_{\text {elastic }}=\frac{E_{0} \times A_{0}}{L_{a}}\left(x+x_{0}\right)
$$

where $A_{0}$ is the original cross-sectional area, $L_{a}$ is the original length of the multi-stacked actuator and $x_{0}$ is the initial pre-compression of the multi-stacked actuator.

The relation between the applied force $F_{0}$ at the tip of the link and $F_{\text {elastic }}$ of the actuator is calculated as follows:

$$
\begin{array}{r}
F_{0}=F_{\text {elastic }} \times \frac{L_{2}}{L} \times \cos \alpha_{1} \times \cos \left(\theta-\alpha_{1}\right) \\
=F_{\text {elastic }} \times \frac{L_{2}}{L} \times \cos \alpha_{1} \times\left(\cos \theta \cos \alpha_{1}+\sin \theta \sin \alpha_{1}\right)
\end{array}
$$

where $L_{2}, L, \alpha_{1}$ and $\theta$ are described in Fig. 10.

Assuming that $\theta, \alpha_{1} \in\left[0, \frac{\pi}{2}\right]$ and define a function $f(x)$ :

$$
\begin{array}{r}
f(x)=\sqrt{\left(L_{0}+x+L_{1}+L_{2}\right)\left(L_{0}+x+L_{1}-L_{2}\right)} \\
\quad \times \sqrt{\left(L_{0}+x-L_{1}+L_{2}\right)\left(-L_{0}-x+L_{1}+L_{2}\right)}
\end{array}
$$

$\sin \theta, \cos \theta, \sin \alpha_{1}$ and $\cos \alpha_{1}$ can be expressed as follows:

$$
\begin{array}{r}
\sin \theta=\cos \left(\frac{\pi}{2}-\theta\right)=\frac{\left(L_{0}+x\right)^{2}+L_{2}^{2}-L_{1}^{2}}{2 L_{2}\left(L_{0}+x\right)} \\
\cos \theta=\sqrt{1-\sin ^{2} \theta}=\frac{f(x)}{2 L_{2}\left(L_{0}+x\right)} \\
\cos \alpha_{1}=\frac{L_{1}^{2}+\left(L_{0}+x\right)^{2}-L_{2}^{2}}{2\left(L_{1}\left(L_{0}+x\right)\right)} \\
\sin \alpha_{1}=\sqrt{1-\sin ^{2} \alpha_{1}}=\frac{f(x)}{2 L_{1}\left(L_{0}+x\right)}
\end{array}
$$

Replacing the results in Eq. 5, the final force at the finger tip is illustrated as follows:

$$
F_{0}=\frac{E_{0} \times A_{0} \times\left(x_{0}+x\right)}{L_{a}} \times \frac{\left(L_{1}^{2}+\left(L_{0}+x\right)^{2}-L_{2}^{2}\right) \times f(x)}{4\left(L_{1}^{2} L\left(L_{0}+x\right)\right)}
$$
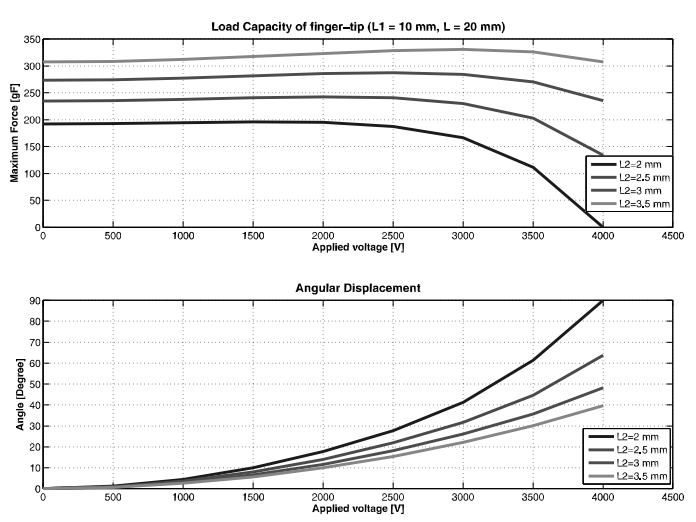

Fig. 11. The simulation results

Based on the analytical equations in Eqs. 2, 3 and 11, the relation between the applied voltage and the force, the applied voltage and the angle of the link are shown in
TABLE I

JOINT DIMENSION

\begin{tabular}{|l||c||c|}
\hline Parameter & Unit & Value \\
\hline Crank length $\left(L_{2}\right)$ & $\mathrm{mm}$ & 2.5 \\
\hline Connecting rod $\left(L_{1}\right)$ & $\mathrm{mm}$ & 10 \\
\hline Young's modulus $\left(E_{0}\right)$ & $\mathrm{MPa}$ & 0.6 \\
\hline Initial area $\left(A_{0}\right)$ & $\mathrm{mm}^{2}$ & 200 \\
\hline Initial length $\left(L_{a}\right)$ & $\mathrm{mm}^{2}$ & 50 \\
\hline
\end{tabular}

11. The requirements of our design are those the link can rotate an angle about $70^{\circ}$ and can lift a mass about $200 \mathrm{~g}$ (the distance from the contact point to the rotational axis is about $20 \mathrm{~mm}$ )at the voltage $4 \mathrm{KV}$. The results in Fig. 11 show the angle and the maximum force of the link at different length of the crank. From the simulation results, it is shown that the length of the crank $2.5 \mathrm{~mm}$ adapts our proposed requirements. Therefore, the dimension of the link is described in Table. I.

\section{Design of one DOF mechanism}

Based on the slider crank mechanism, a one DOF mechanism is designed as illustrated in Fig. 12. The entire link is manufactured from acryl and aluminum. The upper link can move a maximum angle $65^{\circ}$. The implementation of the link is described in Fig. 13. When the driving voltage is applied, the upper link can rotate an angle from $0^{\circ}$ to $65^{\circ}$. The relation between the driving voltage and the angle of the upper link is described in Fig. 14.

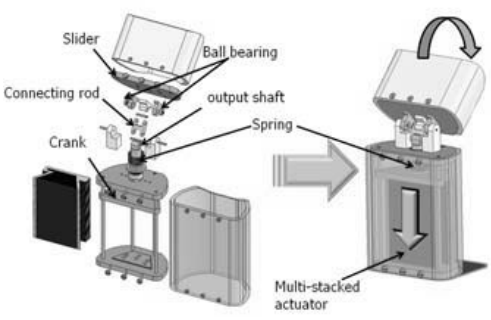

Fig. 12. Configuration of 1-DOF link

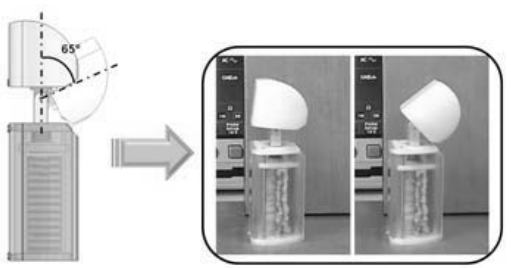

Fig. 13. Implementation of 1-DOF link

\section{DESIGN OF MULTI-JOINT ROBOT FINGER}

Based on the slider crank mechanism and the one DOF mechanism, a multi-joint robot finger utilizing multi-stacked actuator is designed as shown in Fig. 15. The finger includes 


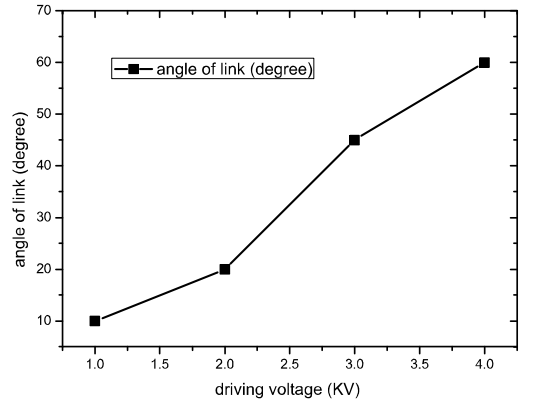

Fig. 14. The relation between the driving voltage and the angle of the link

two DOFs and the entire finger is covered with acrylic material. The lengths of distal middle, and proximal phalanges are $28.3 \mathrm{~mm}, 59 \mathrm{~mm}$ and $72 \mathrm{~mm}$, respectively as detailed in Fig. 16. The total weight of whole finger is $80 \mathrm{~g}$. The prototype of the finger is illustrated in Fig. 18.

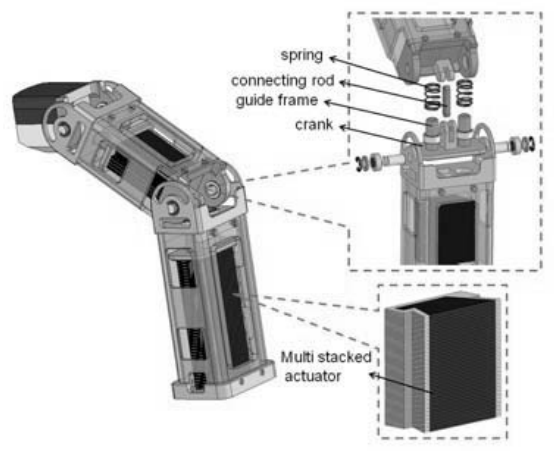

Fig. 15. Configuration of the finger

Also, the joint ranges measured are as follows.

- First joint: $70^{\circ}$

- Second joint: $50^{\circ}$

\section{DESIGN OF THE CONTROLLER}

We developed a low level controller with a circuit completely packaged. The schematic diagram of the controller is shown in Fig. 19. The microcontroller receives the command from the computer through CAN communication. After that, the microcontroller sends the command to high voltage amplifier. Moreover, this microcontroller sends the PWM (Pulse Width Modulation) signals to control high voltage switching circuit to apply the high voltage for multi stacked actuator. The high voltage switching as described in Fig. 20 uses IGBT( IXYS, IXEL25N250) for switching. Because the IGBT can stand the maximum voltage which is $2.5 \mathrm{KV}$, to switch the high voltage until $4 \mathrm{KV}$, two serially connected IGBTs are needed to turn on and turn off the driving voltage of multi-stacked actuator as shown in Fig. 20. The overall circuit is as shown in Fig. 21. The output voltage of the high voltage switch is plotted in Fig. 22. which displays

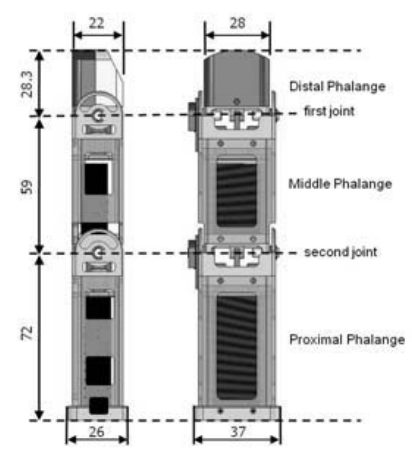

Fig. 16. The dimension of the finger

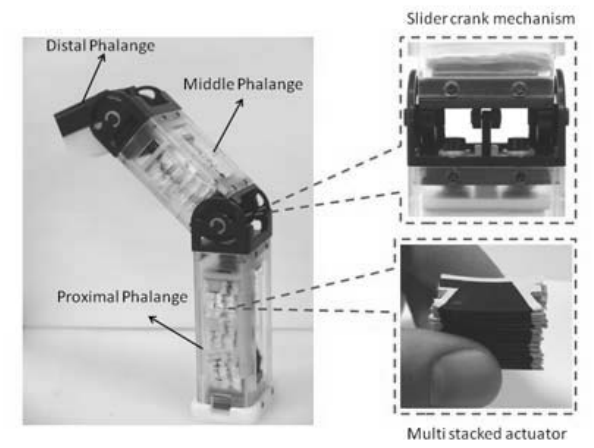

Fig. 17. The real prototype of the finger

the voltage is successfully supplied to the mutlti-stacked actuator.

\section{CONCLUSIONS}

In this paper, a multi-stacked folded actuator based on novel synthetic elastomer is presented. The linear motion generated from the proposed actuator was converted to rotational motion by utilizing a simple slider crank mechanism. By using proposed mechanism, a multi-joint robot finger was built. The advantage of this finger is lightweight and compactness. The controller is useful to drive the finger in high speed motion. In the near future, a multi-joint robot

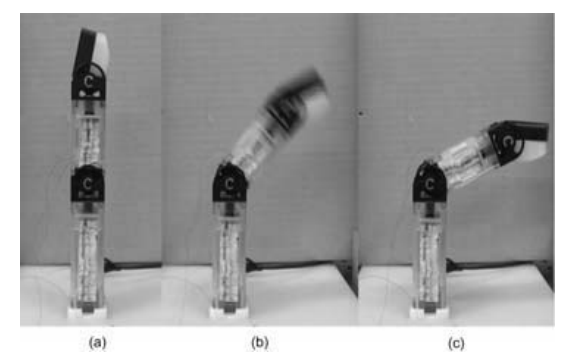

Fig. 18. The operation of the finger 


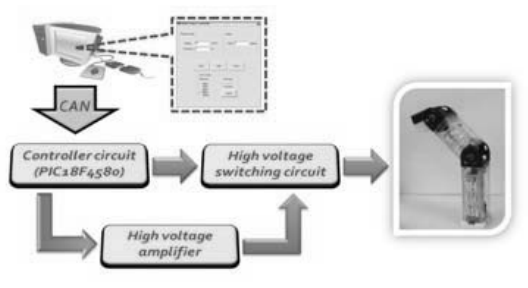

Fig. 19. The diagram of controller

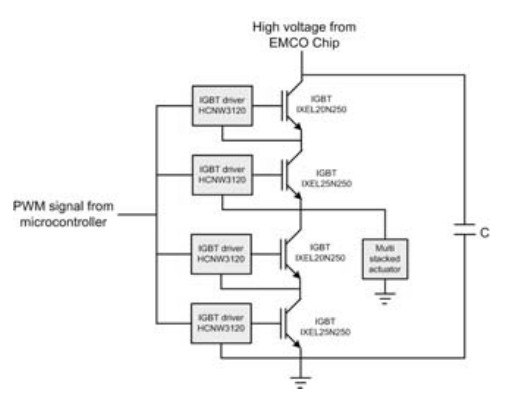

Fig. 20. The high voltage switch diagram

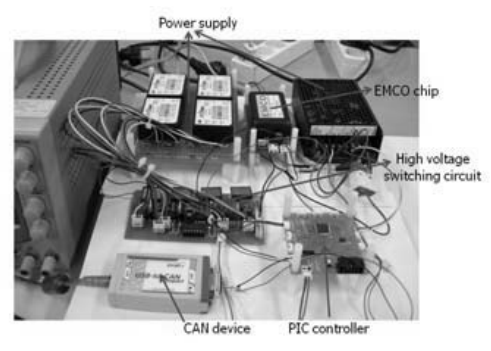

Fig. 21. The circuit to control the finger

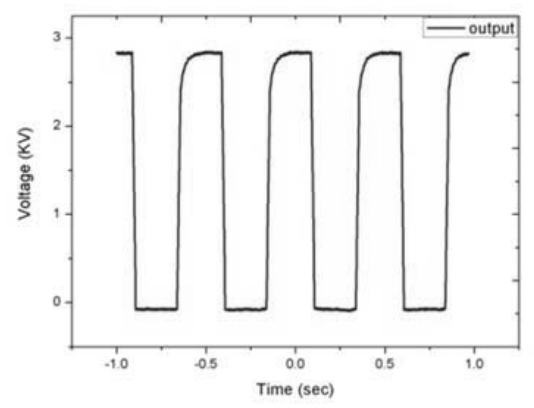

Fig. 22. The output voltage of switch circuit. hand will be developed based on the proposed actuation mechanism.

\section{ACKNOWLEDGMENT}

This research was performed for the Intelligent Robotics Development, one of the 21st Century Frontier R\&D Programs funded by the Ministry of Commerce, Industry and Energy.

\section{REFERENCES}

[1] S. C. Jacoben et al., "The Utah/MIT dexterous hand: Work in progress", Int. J. Robot, Vol. 3, No. 4, pp. 21-50, 1984

[2] J. Butterfass, M. Grebenstein, H. Liu, G. Hirzinger, "DLR-Hand II: Next Generation of a dexterous Robot hand", Proc. of IEEE Int. Conf. on Robotics and Automation, Vol. 1, pp. 109-114, 2001.

[3] H. Kawasaki, T. Komatsu, K. Uchiyama, "Anthropomorphic Robot Hand: Gifu Hand III", Proc. of 2002 Int. Conf. on Control, Automation, and Systems, pp. 1288-1293, 2002.

[4] L. E. Pezzin, T. R. Dillingham, E. J. MacKenzie, P. Ephraim and P. Rossbach, "Use and satisfaction with prosthetic limb devices and related services", Archives of Physical Medicine and Rehabilitation, Vol. 85, pp. 368-375, 2004.

[5] Y. Bar-Cohen, T. Xue, M. Shahinpoor, J. Simpson and J. Smith, "Flexible, low-mass robotic arm actuated electroactive polymer and operated equivalently to human arm and hand", Proc. ASCE Speciality Conf. Robotics for Challenging Environments, pp. 15-21, 1998.

[6] V. Bundhoo and E. J. Park, "Design of an artificial muscle actuated finger towards biomimetic prosthetic hands", International Conference on Advanced Robotics, pp. 368-375, 2005.

[7] A. D. Price, A. Jnifene and H. E. Naguib, "Design and control of a shape memory alloy based dexterous robot hand", Smart Materials and Structures, Vol. 16, pp. 1401-1414, 2007.

[8] K. J. D. Laurentis and C. Mavroidis, "Mechanical design of a shape memory alloy actuated prosthetic hand", Technology and Health Care , Vol. 10, pp. 91-106, 2002.

[9] Y. Bar-Cohen, "Electroactive Polymer (EAP) actuators as artificial muscle", SPIE press, 2nd edition, 2004.

[10] K. J. Kim, and S. Tadokoro, Ed., "Electroactive Polymer for Robotic Applications", Springer, 2007.

[11] R. Pelrine, R. Kornbluh, Q. Pei, J. Joseph, "High-speed Electrically Actuated Elastomer with Strain Greater Than $100 \%$ ", Science, Vol. 287, pp. 836-839, 2000.

[12] Q. Pei, R. Pelrine, S. Stanford, R. Kornbluh, M. Rosenthal, "Electroelastomer rolls and their application for biomimetic walking robots", Synth. Met., Vol. 135-136, pp. 129-131, 2003.

[13] Q. Pei, M. Rosenthal, S. Stanford, H. Prahlad, R. Pelrine, "Multipledegrees-of-freedom electroelastomer roll actuators", Smart Materials and Structures, Vol. 13, pp. N86-N92, 2004.

[14] H. R. Choi, K. M. Jung, S. M. Ryew, J. D. Nam, J. W. Jeon, J. C. Koo, K. Tanie, "Biomimetic Soft Actuator: Design, Modelling, Control and Applications", IEEE/ASME Transaction on Mechatronics, Vol. 10, No. 5, pp. $581-593,2005$.

[15] H. C. Nguyen, J. C. Koo, Y. K. Lee, J. D. Nam, H. R. Choi, "Artificial muscle actuator based on synthetic elastomer", International Journal of Control, Automation and Systems, Vol. 6, 2008.

[16] K. M. Jung, J. C. Koo, J. D. Nam, Y. K. Lee, H. R. Choi, "Artificial annelid robot driven by soft actuators", J. Bioinspiration and Biomimetics, Vol. 2, pp. S42-S49, 2007.

[17] F. Carpi, C. Salaris, D. Rossi, "Folded dielectric elastomer actuators", Smart Materials and Structures, Vol. 16, pp. S300-S305, 2007.

[18] H. F. Shlaak, M. Jungmann, M. Matysek, P. Lotz, "Novel Multilayer Electrostatic Solid-State Actuators with Elastic Dielectric", Proc. Smart Structures and Materials 2005: Electroactive Polymer Actuators and Devices (EAPAD), Vol. 5759, pp. 121-131, 2005.

[19] H. C. Nguyen, J. K. Park, V. T. Doan, H. S. Kim, J. C. Koo, Y. K. Lee, J. D. Nam and H. R. Choi, "Multi-stacked Artificial muscle actuator based on Synthetic elastomer", Proc. IEEE/RSJ Int. Conf. Intelligent Robots and Systems, pp. 771-776, 2007. 